\title{
Aktuelle Studien zu ASS, Depression, Parkinson-Krankheit, Schizophrenie und Stress
}

\author{
Iris Bräuninger
}

$\mathrm{n}$ diesem Beitrag werden aktuelle Studien kurz zusammengefasst. Anhand unterschiedlicher Studientypen wurden verschiedene Krankheitsbilder untersucht: Autismus Spektrum Störung (ASS) und Depression (je ein Systematischer Review mit Metaanalyse), Parkinson-Krankheit (Systematischer Review mit Metaanalyse, 1 RCT), Schizophrenie (MixedMethod mit RCT) und Stress (1 RCT).

\section{Autismus Spektrum Störung (ASS)}

Der Systematische Review mit Metaanalyse von Takahashi et al. (2019) untersuchte die Wirksamkeit von Tanz-, Bewegungstherapie (TT) auf ASS. Aus 3296 Treffern gingen insgesamt sieben Studien über den Zeitraum von 1970 bis 2018 in die Bewertung ein: Zwei wurden beim Level of Evidence (LOE) mit $2 b$ bewertet (Oxford Centre for Evidence-based Medicine 2009), drei mit $3 \mathrm{~b}$ LOE und je eine Studie mit nur 4 LOE beziehungsweise 5 LOE. Die Autorlnnen schlussfolgerten, dass sich das Niveau der TT-Studien zu ASS zwar in den letzten Jahren verbessert habe, zukünftige Forschung jedoch die Aufgabe hätte, „eine größere wissenschaftliche Genauigkeit bei der Dokumentation der Wirksamkeit“ (S.55) von TT-Behandlungsinterventionen nachzuweisen.

\section{Depression}

Der Systematische Review mit Metaanalyse von Karkou et al. (2019) überprüfte TT-RCTs bei Depression. Von 760 Treffern gingen acht Studien in die Datenanalyse ein: An den Studien nahmen $\mathrm{N}=351$ Erwachsene mit leichter bis schwerer Depression an 192 TT-Gruppen teil, während sie wie gewohnt behandelt wurden (treatment as usual - TAU), 159 Erwachsene erhielten nur TAU. Das Ergebnis zeigte die höchste Effektgröße für TT plus TAU bei Erwachsenen mit Depression im Vergleich zu nur TAU. Laut den Autorlnnen wiesen die Studien mäßige bis hohe Qualität auf. Dies interpretierten sie als Indikator dafür, dass TT als wirksame Therapie in der Behandlung von Depression bei Erwachsenen zu bewerten sei.

\section{Parkinson-Krankheit}

Zhang und KollegInnen (2019) überprüften in ihrem Systematischen Review mit Metaanalyse, ob Tanztherapie bei PatientInnen mit Parkinson-Krankheit kognitive und Stimmungssymptome verbesserten im Vergleich zur Kontrollgruppe ohne TT. Von 1178 Studien gingen sieben RCTs in die Berechnung ein. Im Ergebnis zeigte sich die TT gegenüber den Kontrollbedingungen nur zur Verbesserung der 
Exekutivfunktion überlegen, jedoch nicht in der Auswirkung auf die globale kognitive Funktion, Depression und Apathie. Der RCT von Michels et al. (2018) wurde in die zuvor erwähnte Studie von Zhang und KollegInnen (2019) eingeschlossen. Ziel der Studie war zu untersuchen, ob eine einmal wöchentlich stattfindende TT-Intervention über 10 Wochen für Parkinson-PatientInnen sicher, machbar und angenehm wäre, wenn die Interventionen auf die Schwere der Erkrankung abgestimmt seien. Am RCT nahmen lediglich 13 Teilnehmerlnnen teil (neun davon in der TT-Intervention), die Gruppen unterschieden sich allerdings im Alter. Die Ergebnisse können deshalb nur vorläufige Tendenzen aufzeigen, dass TT für das Klientel sicher, machbar und angenehm zu sein scheint.

\section{Schizophrenie}

Ziel der Mixed-Method-Studie mit RCT und halbstrukturierten Interviews war zu überprüfen, ob Tanztherapie plus Standardbehandlung die negativen Symptome bei PatientInnen mit Schizophrenie mehr reduzieren könne als Standardbehandlung allein (Bryl 2018). An der Studie nahmen 31 Personen teil. TT wurde über 10 Wochen zweimal wöchentlich angeboten, im Prä-Test und Post-Test wurden negative Symptome (PANSS, BNSS) und psychosoziale Funktionsweise (WHO-DAS 2.0, SDS) erfasst, welche in der Analyse nur deskriptiv ausgewertet wurden. Im Post-Test zeigte sich ein leichter Anstieg der PANSSWerte in der TT-Gruppe ( $n=15)$, in der Kontrollgruppe $(n=13)$ hingegen eine leichte Abnahme. Die BNSS-Scores verbesserten sich in beiden Gruppen. Die TT-Gruppe berichtete im Vergleich zur Kontrollgruppe über positive Effekte der TT in Bezug auf psychosoziale Funktionen und den Umgang mit negativen Symptomen.

\section{Stress}

Im RCT von Vrinceanu und KollegInnen (2019) wird die Intervention als Tanz-/Bewegungstraining (DMT) beschrieben, es scheint allerdings Tanztherapie gemeint zu sein (bestätigt durch einen Autor). Ziel der Studie war, den Marker für chronischen Stress, das sogenannte Cortisol-Awakening Response (CAR), als Auswirkung auf DMT zu testen. Die vierzig Teilnehmerlnnen (Durchschnittsalter 67,45 Jahre, $75 \%$ Frauen) wurden auf drei Gruppen randomisiert: DMT $(n=12)$, Aerobic-Training (AT, $n=14$ ) und eine Wartegruppe (WG, $n=14)$, wovon DMT und AT dreimal die Woche 60 Minuten über drei Monate hinweg stattfanden. Die Speichelproben wurden an drei aufeinanderfolgenden Tagen zum Vortest und Nachtest erhoben. Als weitere Vor- und Nachtest wurden die Mini-Mental State Examination (MMSE) und fünf standardisierte psychologische Tests durchgeführt (GDS, STAI; SF-12, LSNS-R, PSQI). Im Post-Test zeigte sich der Kortisolwert in der DMT-Intervention signifikant reduziert gegenüber den anderen Gruppen (Gruppen x Zeit Interaktion), welche keine Veränderung gegenüber dem Ausgangswert in ihrer CAR zeigten. Die Herz-Lungen-Fitness verbesserte sich nur in der AT-Gruppe. Die psychologischen Variablen verbesserten sich in keiner Gruppe signifikant.

\section{Literatur}

Bryl, K. (2018): F239. The role of dance / movement therapy in treatment of negative syndrome and psychosocial functioning of patients with schizophrenia: results from a pilot mixed methods intervention study with explanatory intent. Schizophrenia Bulletin 44 (1), 315-316, https://doi.org/10.1093/schbul/sby017.770

Karkou, V., Aithal, S., Zubala, A., Meekums, B. (2019): Effectiveness of dance movement therapy in the treatment of adults with depression: a systematic review with meta-analyses. 
Frontiers in Psychology 10, 936, https://doi. org/10.3389/fpsyg.2019.00936

Michels, K., Dubaz, O., Hornthal, E., Bega, D. (2018): „Dance therapy“ as a psychotherapeutic movement intervention in Parkinson's disease. Complementary Therapies in Medicine 40, 248252, https://doi.org/10.1016/j.ctim.2018.07.005

Oxford Centre for Evidence-based Medicine (2009): Levels of Evidence. In: www.cebm.net/2009/ $06 /$ oxford-centre-evidence-based-medicinelevels-evidence-march-2009, 8.4.2020

Takahashi, H., Matsushima, K., Kato, T. (2019): The effectiveness of dance / movement therapy interventions for autism spectrum disorder: A systematic review. American Journal of Dance Therapy 41 (1), 55-74, https://doi.org/10.1007/ s10465-019-09296-5

Vrinceanu, T., Esmail, A., Berryman, N., Predovan, D., Vu, T. T. M., Villalpando, J. M., Pruessner, J.C., Bherer, L. (2019): Dance your stress away: comparing the effect of dance / movement training to aerobic exercise training on the cortisol awakening response in healthy older adults. Stress 22 (6), 687-695, https://doi.org/10.1080/1025 3890.2019 .1617690
Zhang, Q., Hu, J., Wei, L., Jia, Y., Jin, Y. (2019): Effects of dance therapy on cognitive and mood symptoms in people with Parkinson's disease: a systematic review and meta-analysis. Complementary Therapies in Clinical Practice 36, 12-17, https://doi.org/10.1016/j.ctcp.2019.04.005

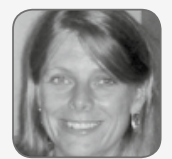

Dr. Iris Bräuninger

Senior Researcher \& Co-Leiterin Studiengang Psychomotoriktherapie (Hochschule für Heilpädagogik Zürich IVE),

Dozentin MA Tanztherapie UAB Barcelona, BTD-Supervisorin / Ausbilderin / Lehrtherapeutin, KMP-Notatorin, Praxis Tanztherapie Supervision Bodensee.

EDr. Iris Bräuninger dancetherapy@mac.com und iris.braeuninger@hfh.ch 